\title{
To Analyze Patients of Post-cholecystectomy Symptoms after Cholecystectomy
}

\author{
Ketan Patel ${ }^{1}$, Jitendra K Mangtani ${ }^{2}$, Neelkamal Gupta ${ }^{3}$, Shavan K Yadav ${ }^{4}$
}

\begin{abstract}
Introduction: Post-cholecystectomy syndrome (PCS) comprises a group of abdominal symptoms that occur after cholecystectomy. Postcholecystectomy syndrome is defined as a group of heterogeneous symptoms. It consists of upper abdominal pain, vomiting, and dyspepsia, which occur after cholecystectomy. Although this term is not accurate, it comprises biliary and non-biliary disorders, possibly not related to cholecystectomy.

Aim: To analyze the clinical evaluation for post-cholecystectomy symptoms.

Objectives: To identify the causes for post-cholecystectomy symptoms. To identify preoperative factors (h/o endoscopic retrograde cholangiopancreatography (ERCP), attacks of cholecystitis) associated with post-cholecystectomy symptoms.

Materials and methods: A prospective clinical hospital-based study was done on 100 patients at a tertiary care referral hospital in the department of general surgery. All cases of post-cholecystectomy who had clinical symptoms of PCS cases in which follow-up ultrasonography (USG) and liver function test (LFT) were done.

Results: In our study, 100 patients were taken who developed PCS after cholecystectomy. Among all patients, 16\% patients developed PCS due to biliary etiology. Among $16 \%$ of patients, the most common cause is recurrent common bile duct (CBD) stone (6\%) and the second most common cause is retained CBD stone (3\%) and sphincter of Oddi dysfunction (3\%).

Discussion: This analysis provides a qualitative overview of etiologies of abdominal symptoms after cholecystectomy. Based on the etiologies of persistent and incident symptoms after cholecystectomy provided in this review, we identified the cause of long-term symptoms after cholecystectomy.

Conclusion: In our study, PCS developed mainly due to non-biliary etiology considered $84 \%$ and biliary etiology considered only $16 \%$. Female has a higher risk to developing post-cholecystectomy symptoms compared to male after cholecystectomy.

Keywords: Cholecystectomy, Common bile duct stone, Post-cholecystectomy syndrome.

Journal of Mahatma Gandhi University of Medical Sciences \& Technology (2020): 10.5005/jp-journals-10057-0136
\end{abstract}

\section{INTRODUCTION}

Post-cholecystectomy syndrome (PCS) comprises a group of abdominal symptoms that occur after cholecystectomy. Postcholecystectomy syndrome is defined as a group of heterogeneous symptoms. It consists of upper abdominal pain, vomiting, and dyspepsia, which occur after cholecystectomy. Although this term is not accurate, it comprises biliary and non-biliary disorders, possibly not related to cholecystectomy. ${ }^{1,2}$

The incidence of post-cholecystectomy symptoms has been reported to be as high as $40 \%$ in one study, and the onset of symptoms may range from 2 days to 25 years. ${ }^{3}$ There may also be gender-specific risk factors for developing symptoms after cholecystectomy. The clinical management of PCS patients is frequently with no evidence-based approach.

Biliary manifestations of PCS usually occur because of operative complications in the early postoperative period, such as bile duct injury/biliary leakage. A late biliary manifestation is commonly caused by inflammatory strictures involving the sphincter of Oddi and the common bile duct, recurrent stone, or biliary dyskinesia. ${ }^{4}$

The most common non-biliary cause of PCS is a peptic ulcer and the most common biliary cause is ductal calculi. Symptomatic treatment is required for a patient who presented with PCS and a complete workup is required to diagnose the actual cause of symptoms; liver function test (LFT) and abdominal ultrasonography (USG) are the early diagnostic tests that are ordered, and further investigations accordingly. 5,6

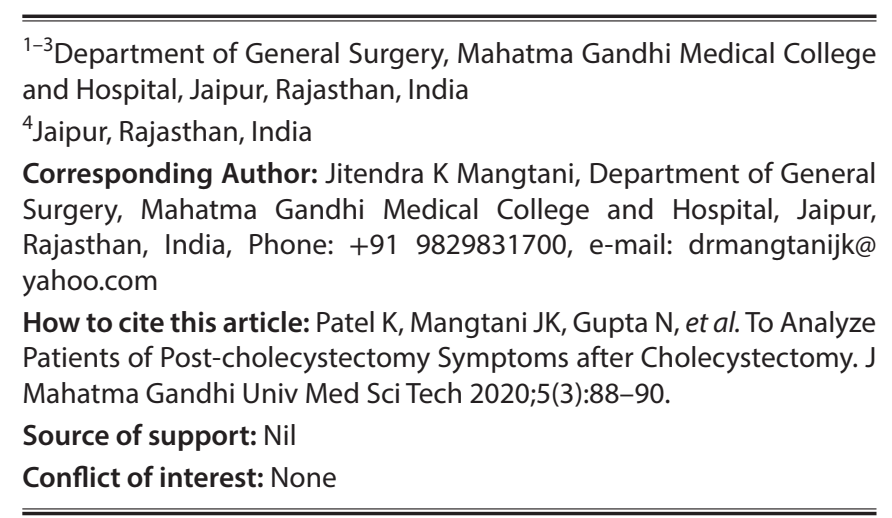

Aıм

- To analyze the clinical evaluation for post-cholecystectomy symptoms.

\section{Objectives}

- To identify the causes for post-cholecystectomy symptoms.

- To identify preoperative factors (h/o endoscopic retrograde cholangiopancreatography (ERCP), attacks of cholecystitis) associated with post-cholecystectomy symptoms.

(c) The Author(s). 2020 Open Access This article is distributed under the terms of the Creative Commons Attribution 4.0 International License (https:// creativecommons.org/licenses/by-nc/4.0/), which permits unrestricted use, distribution, and non-commercial reproduction in any medium, provided you give appropriate credit to the original author(s) and the source, provide a link to the Creative Commons license, and indicate if changes were made. The Creative Commons Public Domain Dedication waiver (http://creativecommons.org/publicdomain/zero/1.0/) applies to the data made available in this article, unless otherwise stated. 
Table 1: Biliary etiology

\begin{tabular}{lcc}
\hline & Number of cases & Percentage \\
\hline Retained CBD stone & 3 & 3.00 \\
Recurrent CBD stone & 6 & 6.00 \\
CBD stricture & 2 & 2.00 \\
Bile leakage & 2 & 2.00 \\
Sphincter of Oddi dysfunction & 3 & 3.00 \\
Total & 100 & 100.00 \\
\hline
\end{tabular}

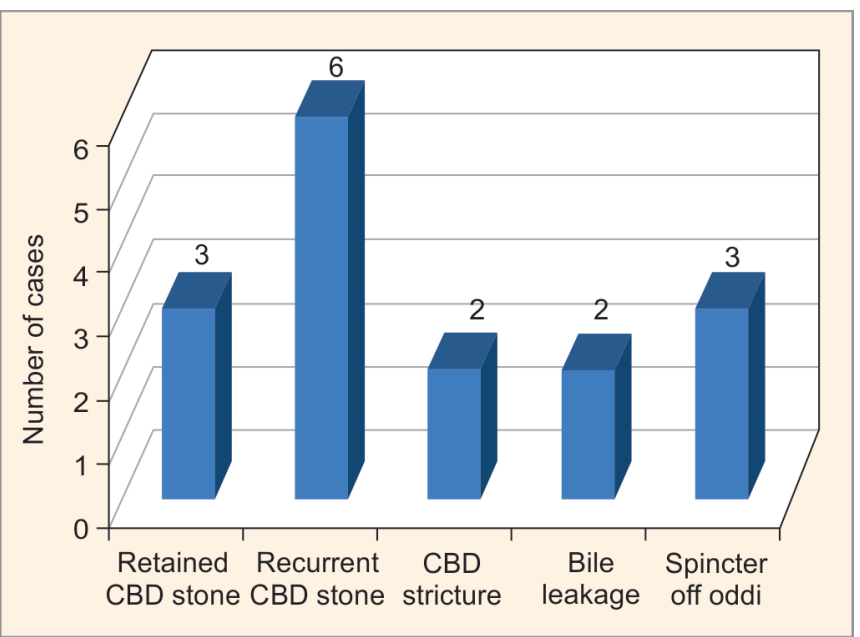

Fig. 1: Biliary etiology

\section{Materials and Methods}

A prospective clinical hospital-based study was done on 100 patients at a tertiary care referral hospital in the department of general surgery.

Study design: Prospective study.

Study period: January 2019 till June 2020.

\section{Inclusion Criteria}

- All cases of post-cholecystectomy who had clinical symptoms of PCS.

- Cases in which on follow-up USG and LFT were done.

\section{Exclusion Criteria}

- Patients in whom LFT and USG were not done.

- Patients who do not give consent for the study.

This study was conducted on 100 patients who developed PCS after cholecystectomy. Patients were chosen randomly irrespective of their age and sex.

All patients have been evaluated with a detailed history of their illness, onset, and duration of presenting symptoms. USG was done in all patients to confirm the diagnosis. All of them were planned to undergo cholecystectomy. Follow-up of patients done on an outpatient basis. Persistent symptoms were diagnosed with USG abdomen, LFT, and ERCP.

Appropriate statistical tests will be applied to find a significant association. $p$ value $<0.05$ will be considered statistically significant.

\section{Results}

In our study, 100 patients were taken who developed PCS after cholecystectomy. Among all patients, $16 \%$ of patients developed
Table 2: Non-biliary etiology

\begin{tabular}{lcc}
\hline & Number of cases & Percentage \\
\hline Gastritis & 42 & 42.00 \\
Pancreatitis & 2 & 2.00 \\
Peptic ulcer & 18 & 18.00 \\
No obvious cause & 22 & 22.00 \\
Total & 100 & 100.00 \\
\hline
\end{tabular}

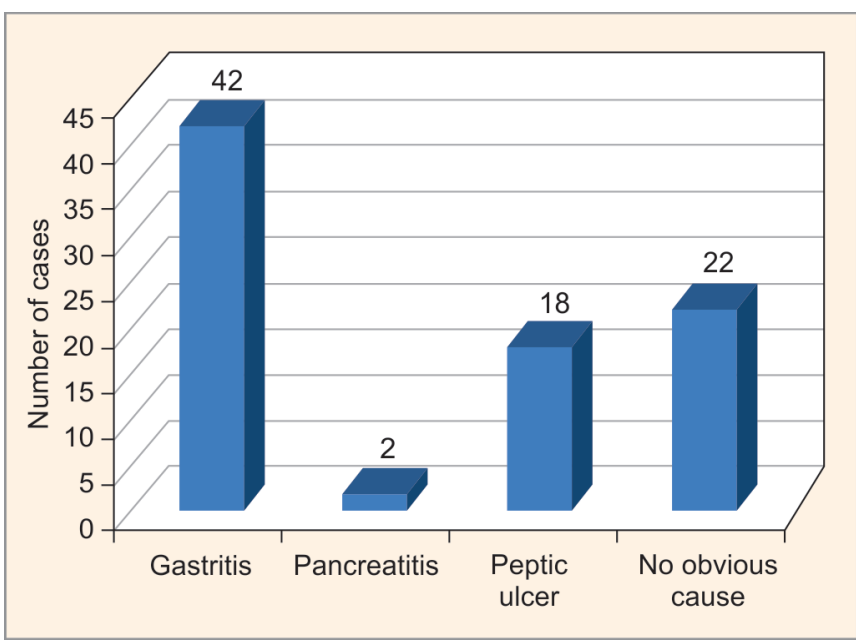

Fig. 2: Non-biliary etiology

PCS due to biliary etiology. Among $16 \%$ of patients, the most common cause is recurrent CBD stone (6\%), and the second most common cause is retained common bile duct (CBD) stone (3\%) and sphincter of Oddi dysfunction (3\%) (Table 1 and Fig. 1).

- $84 \%$ of patients developed PCS due to non-biliary etiology, among $84 \%$ of patients most common cause is gastritis (42\%) which shows that most of the post-cholecystectomy symptoms developed from non-biliary etiology.Among all patients, 42 were diagnosed with gastritis, 18 patients were diagnosed with peptic ulcer, and 2 patients were diagnosed with pancreatitis and they were managed conservatively (Table 2, and Figs 2 and 3 ).

- Among 100 patients, 41 patients are those who do not have any obvious risk factor, 23 patients have previous h/o ERCP, 16 patients have the previous attack of cholecystitis, and 15 patients have obesity who developed PCS (Table 3).

\section{Discussion}

This analysis provides a qualitative overview of etiologies of abdominal symptoms after cholecystectomy. Based on the etiologies of persistent and incident symptoms after cholecystectomy provided in this review, we identified the cause of long-term symptoms after cholecystectomy.

In our study, we categorized the etiology of long-term postoperative symptoms after cholecystectomy. We established that most persisting symptoms are likely to be caused by non-biliary etiology as compared to biliary etiology. Detailed anamnesis and tailored diagnostic tests (such as ultrasound, ERCP, LFT, pancreatic marker, and upper GI endoscopy) will provide insight into the presence of abdominal disorders.

Analysis of our cohort during follow-up showed that there were two constant factors (previous attacks of acute 


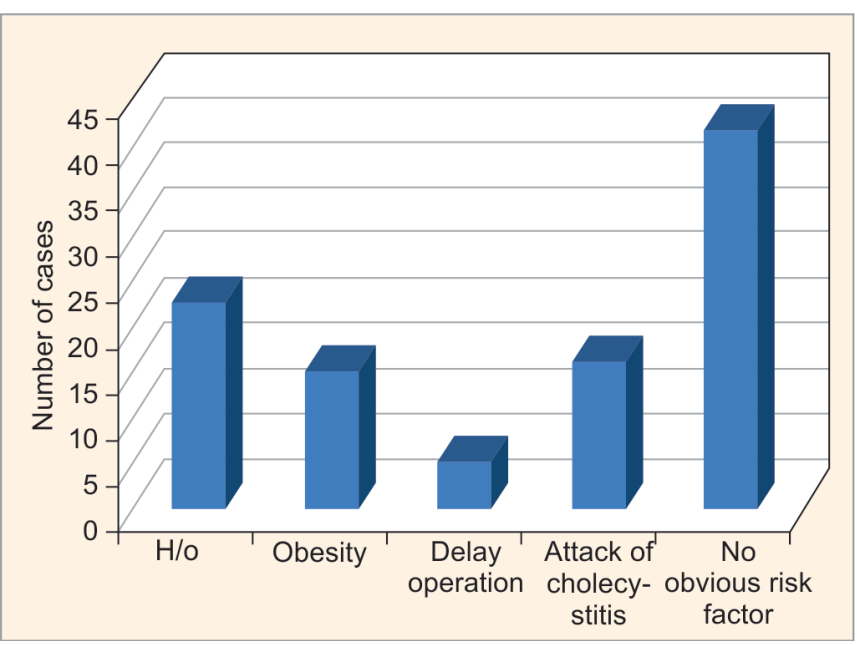

Fig. 3: Risk factor

Table 3: Risk factor

\begin{tabular}{lcc}
\hline & Number of cases & Percentage \\
\hline H/o ERCP & 23 & 23.00 \\
Obesity & 15 & 15.00 \\
Delay operation & 5 & 5.00 \\
Attack of cholecystitis & 16 & 16.00 \\
No obvious risk factor & 41 & 41.00 \\
Total & 100 & 100.00 \\
\hline
\end{tabular}

cholecystitis and previous h/o ERCP) that significantly affected the development of PCS. Prior attacks of acute cholecystitis have been associated with PCS and the mechanism by the theory of referred pain from the gallbladder which continuing or manifesting postoperatively.

In a study conducted by Jaunoo et al., ${ }^{7}$ bile duct injury is seen $0.4-4 \%$, and retained/recurrent CBD stone is seen $1.2-14 \%$.

In our study, we had taken all follow-up, 100 patients of abdominal pain, after cholecystectomy. Among all patients, six patients were diagnosed with recurrent CBD stone, three patients were diagnosed with retained CBD stone, two were diagnosed with bile leakage, and two were diagnosed with CBD stricture.
In a study conducted by Murshid et al., ${ }^{8}$ the most common cause of PCS is extrabiliary disorders, such as, gastritis, peptic ulcer, irritable bowel syndrome, or pancreatitis ranging from 1 to $75 \%$.

In our study, we had taken all follow-up, 100 patients of abdominal pain, after cholecystectomy and they were evaluated by LFT, a pancreatic enzyme, USG, ERCP, and upper GI endoscopy. Among all patients, 42 were diagnosed with gastritis, 18 patients were diagnosed with peptic ulcer, and 2 patients were diagnosed with pancreatitis and they were managed conservatively. Those patients who had PCS due to biliary etiology were managed by ERCP.

\section{Conclusion}

In our study, PCS developed mainly due to non-biliary etiology considered $84 \%$, and biliary etiology considered only $16 \%$. Female has a higher risk to developing post-cholecystectomy symptoms compared to male after cholecystectomy. Among all causes, gastritis is the most common cause of developing PCS. Patients who were at higher risk of developing PCS, had previous h/o ERCP, attacks of cholecystitis, and obesity. Half of the patients who develop PCS has no identified previous risk factor.

\section{References}

1. Schofer JM. Biliary causes of postcholecystectomy syndrome.J Emerg Med 2010;39(4):406-410. DOI: 10.1016/j.jemermed.2007.11.090.

2. Terhaar OA, Abbas S, Thornton FJ, et al. Imaging patients with "postcholecystectomy syndrome": an algorithmic approach. Clin Radiol 2005;60(1):78-84. DOI: 10.1016/j.crad.2004.02.014.

3. Yamada T, ed. Text Book Gastroenterology. 2nd ed., Philadelphia: Lippincott; 1995.

4. Zhou PH, Liu FL, Yao LQ, et al. Endoscopic diagnosis and treatment of postcholecystectomy syndrome. Hepatobiliary Pancreat Dis Int 2003;2:117-120.

5. Girometti R, Brondani G, Cereser L, et al. Post-cholecystectomy syndrome: spectrum of biliary findings at magnetic resonance cholangiopancreatography. Br J Radiol 2010;83(988):351-361. DOI: 10.1259/bjr/99865290.

6. Arora D, Kaushik R, Kaur R, et al. Post-cholecystectomy syndrome: a new look at an old problem. Access Surg 2018;14(3):202-207. DOI: 10.4103/jmas.JMAS_92_17.

7. Jaunoo SS, Mohandas S, Almond LM. Postcholecystectomy syndrome (PCS). Int J Surg 2010;8(1):15-17. DOI: 10.1016/j.ijsu.2009.10.008.

8. Murshid KR. The post cholecystectomy syndrome: a review. Saudi J Gastroenterol 1996;2:124-137. 